\title{
Topology optimization of Piezoelectric Macro Fiber Composite Patches on Composite Plates for active vibration
}

\section{control}

\author{
Eduardo Padoin ; Jun Sergio Ono Fonseca ${ }^{2}$; Odair Menuzzi ${ }^{3}$ \\ Department of Mechanical Engineering, UFRGS, Porto Alegre, RS,
}

\begin{abstract}
This paper presents a topology optimization methodology of piezoelectric actuators in laminated composite structures. The Linear Quadratic Regulator (LQR) optimal control technique is used. The topology optimization is formulated to find the optimum localization of the Macro Fiber Composite (MFC), through of the maximization of the controllability index. Actuator location optimization results show that the technique implemented improves the structural vibration damping.
\end{abstract}

Keywords. Topology optimization, macro fiber composite, LQR optimal control, laminated composite material

\section{Introduction}

The increasing demand for light structures in important applications highlights the necessity for using advanced methods in their design, as the structural optimization and optimal control. With sensors and actuators integrated by a control system, these structures have the capacity of sense environment changes, diagnose localized problems, store and process measurement data; then they take appropriate action to improve the efficiency of the system, or to preserve their structural integrity and safety [3]. An important application, according to [5], are systems that can sense induced motions and, thus, apply control force to reduce the structural vibrations. The main application of these smart structures is in the aerospace industry, where they can be seen in flexible robot manipulators [18].

The development of methods to design smart structures is a promising research field, fueled by the existing demand for these structures and the great range of possible applications. In the realm of the structural design, the topology optimization method contributed efficiently in the design of lighter structures, decreasing costs and the material utilized, evidencing the sustainability aspects [7,2].

Optimized structures usually present reduced mass and low internal damping. These features facilitate the occurrence of structural vibrations, which may cause undesirable operational effects, for instance in mechanism positioning accuracy. According to [13], the use of an active control system integrated by sensors and actuators is crucial in smart

\footnotetext{
1 eduardopadoin@bol.com.br

2 jun@ufrgs.br

3 odair.menuzzi@ibest.com.br
} 
structures. This article presents the application of a specific type of actuator, the MFC (Macro Fiber Composite) piezoelectric transducer [15].

This work uses a simplified model to represent the interaction between the MFC and the structure. Most works models the electromechanical effect, where it is necessary to consider in this model the electric field generated between the electrodes, the piezoelectric constitutive model coupling mechanical and electrical fields, resulting thus in a complex multi-physic model [11,4].

Based on the aforementioned ideas, this work presents a topology optimization of piezoelectric actuators on laminated composite structures, aiming to reduce the structural vibrations induced by external forces. The Linear Quadratic Regulator (LQR) control is used for the structural control. The dynamic system is represented in state space using modal coordinates $[12,13,6]$. In the active control of structural vibrations using piezoelectric material, the localization of sensors and actuators has influence in performance of the control system [9]. Therefore, the work presents a topology optimization that allows the optimal MFC placement by the maximization of controllability index.

\section{Structural Modeling}

The approximated solution of the solid mechanics equilibrium equations uses finite element method (FEM) formulation. It uses the assumptions of the infinitesimal linear elasticity and a lamination theory for composite materials. Mass and stiffness matrices are derived for an eight-node serendipity quadrilateral shell element. The element is based on the Ahmad degenerated solid formulation [1], and it has five degrees-of-freedom (three displacements and two slopes) per node. A good review of the element can be found in [17]. The laminated composite formulation uses an explicit integration through the thickness.

\subsection{First-order shear deformation laminated theory}

The First Order Deformation Laminated Theory (FSDT) is based on the ReissnerMindlin plate theory. It allows the inclusion of transverse shear strains assuming a linear variation through the thickness [14]. In this theory, the constitutive equation of laminated plate is described as:

$$
\left\{\begin{array}{l}
\boldsymbol{N} \\
\boldsymbol{M}
\end{array}\right\}=\left[\begin{array}{ll}
\boldsymbol{C}_{1} & \boldsymbol{C}_{2} \\
\boldsymbol{C}_{2} & \boldsymbol{C}_{3}
\end{array}\right]\left\{\begin{array}{l}
\varepsilon \\
\kappa
\end{array}\right\},
$$

where $N$ and $M$ are the distributed normal tractions and moments applied to the plate, respectively. The $\varepsilon$ are the midplane (membrane) strains terms, and $\kappa$ are the curvatures terms.

The matrices $\boldsymbol{C}_{i}$ are standard in finite element shell modeling, and are defined generically by

$$
\boldsymbol{C}_{i}=\frac{1}{i} \sum_{k=1}^{n} \boldsymbol{Q}_{k}\left(\boldsymbol{Z}_{k+1}^{i}-\boldsymbol{Z}_{k}^{i}\right), \quad i=1,2,3
$$

where $z$ is the distance from laminate midplane to the bottom of the each $k$-th layer, $Q_{k}$ is the transformed stiffness matrix and $n$ is the number of layers.

$C_{1}$ is the extensional stiffness matrix; the stiffness matrix $C_{2}$ is the coupling between 
bending and membrane, which contains the contribution of the curvature $\kappa$ in the traction, when the plate is symmetric it is zero. The matrix $C_{3}$ is the bending stiffness matrix. Whereas the derivation of the transformation matrices and their many curvilinear coordinate systems is quite involved, it is possible find a good review about the formulation presented in this section, in composite material shell books [14].

\subsection{Stiffness and mass matrices}

The stiffness matrix utilized in this work follows a formulation proposed by [10], using an explicit integration on the thickness direction. The elemental transverse integration is accomplished using the third model presented in that paper, in which the inverse isoparametric mapping Jacobian matrix is assumed constant, and its computations are carried only on the reference surface.

Thus the stiffness matrix can be described as

$$
\boldsymbol{K}_{e}=\int_{\Omega_{e}} \boldsymbol{F}^{T} \boldsymbol{C F} d \Omega_{e}
$$

where $K_{e}$ is elementary stiffness matrix, and the strain matrix $F$ is divided in two parts, both are independent of the thickness and can be rewritten as

$$
\boldsymbol{F}=\boldsymbol{F}_{1}+z \boldsymbol{F}_{2} .
$$

Using (4) the elementary stiffness matrix can be written as

$$
\boldsymbol{K}_{e}=\int_{-1}^{1} \int_{-1}^{1}\left[\boldsymbol{F}_{1}^{T} \boldsymbol{C}_{1} \boldsymbol{F}_{1}+\boldsymbol{F}_{1}^{T} \boldsymbol{C}_{2} \boldsymbol{F}_{2}+\boldsymbol{F}_{2}^{T} \boldsymbol{C}_{2} \boldsymbol{F}_{1}+\boldsymbol{F}_{2}^{T} \boldsymbol{C}_{3} \boldsymbol{F}_{2}\right]|J| d \eta d \xi,
$$

where the coordinates $\xi$ and the Jacobian matrix $\mathbf{J}$ arise from the standard isoparametric coordinate mapping [17].

This work uses the lumped mass matrix [17]. Global stiffness and mass matrices are then assembled by a conventional FEM superposition procedure.

\subsection{Distributed force vector due to the MFC}

This work uses a simplified model to represent the interaction between the MFC actuator and the structure. This model has the advantage a simpler implementation, avoiding modeling electric fields and the electromechanical effect. Assuming that the MFC is modeled as one of the orthotropic material layers of the laminate shell, it is considered that an additional elemental force vector of distributed loads is obtained from an initial strain in the MFC. This strain is proportional to the applied electrical potential. Therefore, is not necessary to explicitly model the electric field and the electromechanical coupling because these effects are considered analytically.

The force vector of distributed loads on the shell surface can be obtained by the following equation

$$
\varepsilon^{0}=\varepsilon_{M F C} \phi
$$

where $\boldsymbol{\varepsilon}_{M F C}$ is the MFC strain vector and $\phi$ is the electric potential.

The strain vector is represented by 


$$
\boldsymbol{\varepsilon}_{M F C}=\left(\varepsilon_{x x}^{M F C},-v_{12} \varepsilon_{x x}^{M F C}, \varepsilon_{z z}^{M F C}, 0,0,0\right),
$$

where $\varepsilon_{z Z}^{M F C}$ is obtained considering the lamina membrane stress state for orthotropic material. The MFC free strain in direction $x$ is obtained from the manufacturer data sheet.

After that, multiplying of the initial strain (7) by laminate stiffness matrix $C$ yields the mechanical stress generated for this strain. Multiplying by interpolation functions matrix $N$ and integrating on the element, it is possible to find the nodal forces values for this element,

$$
\boldsymbol{f}_{d}=\int_{\Omega_{\mathrm{t}}} \boldsymbol{\varepsilon}^{0^{T}} \boldsymbol{C} \boldsymbol{F} d \Omega_{\mathrm{t}}
$$

so the distributed force vector in the surface each element can be written as

$$
\boldsymbol{f}_{d}=\int_{-1}^{+1} \int_{-1}^{+1}\left[\boldsymbol{\varepsilon}^{0^{T}} \boldsymbol{C}_{1} \boldsymbol{F}_{1}+\boldsymbol{\varepsilon}^{0^{T}} \boldsymbol{C}_{2} \boldsymbol{F}_{2}\right]|J| d \xi d \eta
$$

Depending on the position of the MFC layer in the stack, and the curvature, the elemental force vector can contain only membrane contributions, or more likely also bending moments and shear forces.

\section{Results}

\subsection{Topology optimization}

In order to find the optimal placement of the MFC actuator to control the structural vibrations of a 80x420x1mm glass-epoxy plate controlled with Smart Material MFC model 8528-P1, a strategy based in the maximization of the controllability index is applied. The objective function is the trace of the controllability Gramian and topology optimization formulation can be described as:

$$
\begin{gathered}
\max _{\rho_{a}} f_{a}=\operatorname{tr}\left(\boldsymbol{W}_{c}\right), \\
\text { subject to }\left\{\begin{array}{c}
0<\rho_{a i} \leq 1, \quad(i=1,2, \ldots, N), \\
V_{a}=\frac{\int_{\Omega} \rho_{a i} d \Omega}{\int_{\Omega} d \Omega} \leq V_{a}^{\max },
\end{array}\right.
\end{gathered}
$$

where $\rho_{a}$ is the control design variable, $V_{a}^{\max }$ is the maximum value of the volume constraint, $\mathrm{N}$ is the vector dimension of the design variable. The controllability Gramian matrix $\boldsymbol{W}_{C}$ of the system is defined by the following Lyapunov equation [13]:

$$
A W_{C}+W_{C} A^{T}+B B^{T}=0
$$

where A and B are the matrices defined in [6]. In this work the trace of the Gramian is used as a measure of controllability of the system [19]. In the solution of the topology optimization problem the Sequential Linear Programming SLP was used.

The Figure 1 presents the optimal MFC placement, where it maximizes the controllability index. 


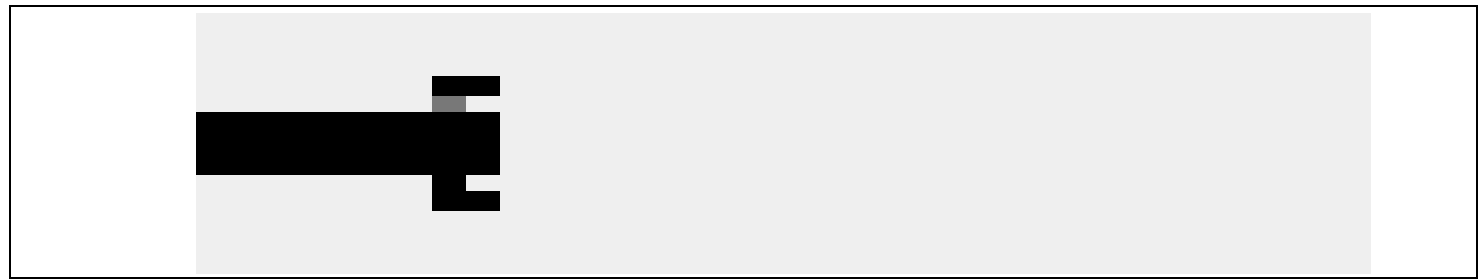

Figure1: Optimal placement for the first bending mode.

In the Figure 1 the optimal placement is localized in the clamped tip. The optimal topology is associated with the region of greater bending moment in the structure for the first vibration mode, and consequently, the region where the greatest controllability index is obtained.

The Figure 2 shows the MFC optimal placement on the active layer for controlling first two vibration modes.

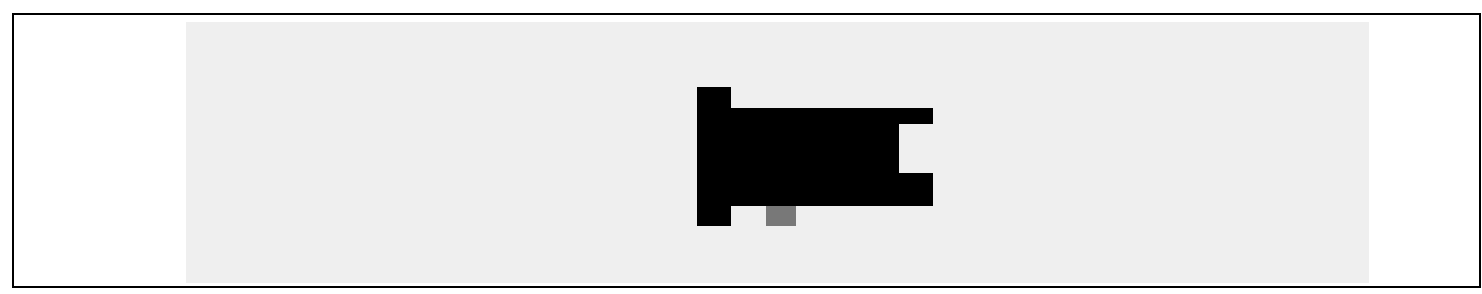

Figure 2: Optimal placement for two bending modes.

In the Figure 2 it is possible observe that the MFC optimal placement is localized in the middle of the structure. It happens due the influence of the second vibration mode. The optimal topology is associated with the region of greater bending moment in the structure for the second vibration mode, and consequently, the region where the greatest controllability index is obtained.

\subsection{Behavior of the Controlled Structure}

The control is designed to reduce structural vibrations, decreasing the system overshoot and settling time to an initial displacement of $10 \mathrm{~mm}$ with null initial velocity in the plate free tip. A truncated modal structural model with the five first vibration modes is considered. A structural damping of 0.018 was obtained experimentally by the authors by applying the logarithm decrement [12].

To determine the values of the weighting matrices ( $\mathrm{Q}$ and $\mathrm{R})$, it was established as choice criterion, the voltage limit supported by MFC, between -500 and 1500 volts, avoiding thus the depolarization of the material. The value is $10^{8}$ times the $2 \times 2$ identity matrix.

The transverse displacement in free tip was evaluated considering the system in closed loop and open. Figure 3 shows the results of the transverse displacement and the applied control signal to the first bending mode. The LQR control system in closed loop contributes to decrease the free tip displacement. The overshoot and the settling time are reduced significantly. The maximum value to electric stress applied was 650 volts and the minimum value was -487 volts keeping within the actuator operational limits. 


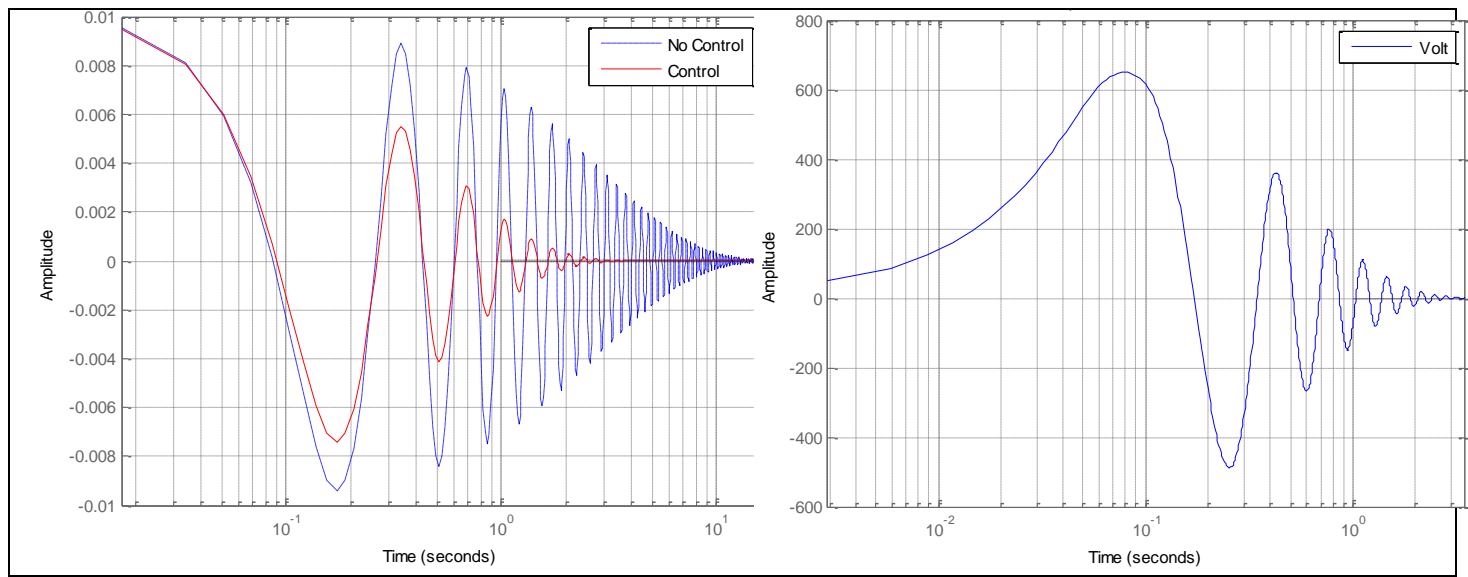

Figure 3: Transverse displacement of free tip (m) and control signal (V) to the model to the first bending mode.

\section{Conclusions}

The topology optimization procedure proposed in this article increases the MFC actuation power to control the structural vibrations. The optimization formulated for the maximization of the controllability index. In the control analysis is possible see that the LQR control system in closed loop contributes to decrease the displacement in free tip. The overshoot and the settling time are reduced significantly. Besides, by a sensible choice of the gain values, the maximum and minimum value to electric potential applied was within of MFC operating limits.

\section{Acknowledgments}

The authors thank CAPES and UFGRS for financial aid.

\section{References}

[1] S. Ahmad, B.M. Irons, and O.C. Zienckievicz. Analysis of Thick and Thin Shell Structure by Curved Finite Elements, Int. J. for Numerical Methods in Engineering, v. 2, p. 419-451, (1970).

[2] M.P. Bendsøe and O. Sigmund. Topology Optimization - Theory, Methods and Applications. Berlin: Springer, (2003).

[3] F.Y. Cheng, H. Jiang, and K. Lou. Smart Structures: Innovative Systems for Seismic Response Control, Boca Raton: CRC, (2008).

[4] M. Collet, M. Ruzzene, K. Cunefare, and B. Xu. Modeling and Characterization of Macro-Fiber Composite Transducers for Lamb Wave Excitation, in SPIE "Health Monitoring of Structural and Biological Systems", (2010). 
[5] E.F. Crawley. Intelligent Structures for aerospace: A technology overview and assessment, AIAA Journal, v. 32. p. 1689-1699, (1994).

[6] W. Gawronski. Advanced Structural Dynamics and Active Control of Structures, New York: Springer, (2004).

[7] R. Haftka, and Z. Gürdal. Elements of Structural Optimization. Dordrecht: Kluwer, 3rd ed, (1991).

[8] A. Kovalovs, E. Barkanov, and S. Gluhihs. Active control of structures using macrofiber composite (MFC), J. Physics: Conference Series v. 93, (2007).

[9] R. Kumar and S. Narayanan. Active vibration control of beams with optimal placement of piezoelectric sensor/actuator pairs, Smart Materials and Structures, v. 17, p. 01-15, (2008).

[10] W.P.P. Kumar and R. Palaninathan. Finite Element Analysis of Laminated Shells with Exact Through-Thickness Integration, Computers and Structures, v. 63(1), p.173-184, (1997).

[11] J. Latalski. Modeling of MFC piezoelectric active elements in ABAQUS system, Maintenance \& Reliability, v.4 p. 72-78, (2011).

[12] L. Meirovitch. Dynamics and Control of Structures, New York: Wiley, (1990).

[13] A. Preumont. Vibration Control of Active Structures, An Introduction. Dordrecht: Kluwer, (2002).

[14] J.N. Reddy, "Mechanics of Composite Plates and Shells - Theory and Analysis". New York: Wiley, (2004).

[15] Smart Material, http://www.smart-material.com. Internet, March (2011).

[16] Y.X. Zhang and Z.H. Yang. Recent Developments in Finite Element Analysis For Laminated Composite Plates, Composite Structures, v. 88, p. 147-157, (2009).

[17] O.C. Zienkiewicz, and R.L. Taylor. The Finite Element Method - Solid Mechanics, Oxford: Butterworth-Heinemann, (2000).

[18] D.G. Zimcik, and A. Yousefi-Koma. Applications of Smart Structures to Aircraft for Performance Enhancement, Canadian Aeronautics and Space Journal, v. 49, p. 163-172, (2003). 